\title{
The Electric Dipole Form Factor of the Nucleon
}

\author{
W.H. Hockings ${ }^{1}$ and U. van Kolck ${ }^{2}$ \\ Department of Physics \\ University of Arizona \\ Tucson, AZ 85721
}

\begin{abstract}
The electric dipole form factor of the nucleon stemming from the QCD $\bar{\theta}$ term is calculated in chiral perturbation theory in leading order. To this order, the form factor originates from the pion cloud. Its momentum dependence is proportional to a non-derivative time-reversal-violating pion-nucleon coupling, and the scale for momentum variation - appearing, in particular, in the radius of the form factoris the pion mass.
\end{abstract}

\footnotetext{
${ }^{1}$ hockings@physics.arizona.edu

${ }^{2}$ vankolck@physics.arizona.edu
} 
Electric dipole moments (EDMs) of the neutron and of atoms have long been of interest because precise experiments are possible, providing strict bounds on possible sources of $C P$ violation. (For a review of both experimental and theoretical results, see for example Ref. [1].) New experiments with ultracold neutrons in preparation at various laboratories (LANL, PSI, ILL, München) promise to provide even higher precision for the neutron EDM than the current value $d_{n}=(-1.0 \pm 3.6) \cdot 10^{-26} e \mathrm{~cm} \mathrm{[2]}$. While neutron experiments yield bounds on the neutron EDM directly, atomic effects are sometimes sensitive to the nuclear Schiff moment, which in turn receives a contribution from the radius of the nucleon electric dipole form factor (EDFF).

Nucleon properties are notoriously difficult to calculate directly from QCD. At low momenta, $Q \sim m_{\pi} \ll M_{Q C D}$, where $m_{\pi}$ is the pion mass and $M_{Q C D} \sim 1 \mathrm{GeV}$ is the typical mass scale in QCD, they can nevertheless be described in terms of an effective field theory (EFT) involving nucleons and pions (and delta isobars), known as chiral perturbation theory $(\chi \mathrm{PT})$. (For an introduction, see for example Refs. [3,4].) In this theory, long-range effects due to the light pions are separated from short-range effects due to all higher-energy degrees of freedom. Observables are systematically expanded in powers of $Q / M_{Q C D}$ (times functions of $Q / m_{\pi}$ ). The pion cloud around the nucleon can generate contributions that are non-analytic in momenta and quark masses, and are calculable in terms of pion and nucleon (and delta) parameters. In contrast, shortdistance physics contributes powers of $Q^{2}$ and $m_{\pi}^{2}$ that can only be calculated using detailed knowledge of the QCD dynamics.

The leading non-analytic contribution to the nucleon EDM coming from the QCD $\bar{\theta}$ angle was first calculated in $\chi \mathrm{PT}$ in Ref. [5], and rederived many times since, see e.g. Ref. [6]. A measurement of the neutron EDM cannot disentangle this long-range effect from short-range, analytic terms. Since a cancellation between different functions of $m_{\pi}^{2}$ is unlikely, the leading non-analytic contribution serves as an estimate of the EDM.

Here we consider the full nucleon EDFF in leading order in $\chi \mathrm{PT}$. The other nucleon form factors have all been calculated previously in this theory at the first few orders: electric [7], magnetic [7], and anapole [8]. Needless to say, the momentum dependence of the EDFF is not as easily accessible experimentally. However, it might become of interest after the neutron EDM (the EDFF at $Q^{2}=0$ ) is measured. As we will show below, the EDFF is in leading order a calculable isovector function of $Q^{2} / m_{\pi}^{2}$, entirely determined by the pion cloud. It is $m_{\pi}^{2}$ that determines the scale of variation in the form factor - an example of a low-energy theorem. In particular, the radius of the EDFF is fixed by $m_{\pi}^{2}$, as first derived in Ref. [9]. If measured, the neutron EDFF would allow a determination of the $T$-violating pion-nucleon coupling (within the precision of the $\chi \mathrm{PT}$ expansion).

At $Q \sim m_{\pi}$, the nucleon is essentially non-relativistic, because $m_{N} \sim M_{Q C D}$. Pions must explicitly be accounted for in the theory, since they are the light (pseudo-)Goldstone bosons corresponding to the spontaneous breaking of (explicitly-broken) chiral $S U_{L}(2) \times$ $S U_{R}(2) \sim S O(4)$ symmetry down to $S U_{L+R}(2) \sim S O(3)$. Also, since the mass difference between the $\Delta$ isobar and the nucleon is comparable to the pion mass, $m_{\Delta}-m_{N} \sim$ $2 m_{\pi}$, the $\Delta$ should be included as well (which is not much of a problem since at these momenta, the $\Delta$ is also a nonrelativistic object). This can be done by first constructing 
the most general Lagrangian involving nucleons, pions, and deltas that transforms under the symmetries of QCD in the same way as QCD itself. Along with this, one needs a power-counting scheme so that interactions can be ordered according to the expected size of their contributions. The strong-interaction Lagrangian contains an infinite number of terms that can be grouped according to the index $\Delta$ :

$$
\mathcal{L}=\sum_{\Delta=0}^{\infty} \mathcal{L}^{(\Delta)} \quad, \quad \Delta \equiv d+f / 2-2
$$

where $d$ is the number of derivatives, powers of $m_{\pi}$ and/or powers of $m_{\Delta}-m_{N}$, and $f$ is the number of fermion fields. Electromagnetic interactions are proportional to the small charge $e$, and it is convenient to account for factors of $e$ by enlarging the definition of $d$ accordingly.

The technology for constructing the Lagrangian is well known, see for example Ref. [3]. We will need the following $T$-conserving terms, which either obey chiral symmetry or break it in the same way as the quark-mass terms do:

$$
\mathcal{L}_{\text {str } / e m}^{(0)}=\frac{1}{2} D_{\mu} \boldsymbol{\pi} \cdot D^{\mu} \boldsymbol{\pi}-\frac{1}{2 D} m_{\pi}^{2} \boldsymbol{\pi}^{2}+\bar{N} i v \cdot D N-\frac{g_{A}}{f_{\pi}} \bar{N}(S \cdot D \boldsymbol{\tau} \cdot \boldsymbol{\pi}) N+\ldots
$$

Here $\boldsymbol{\pi}$ denotes the pion field in a stereographic projection of $S O(4) / S O(3)$, with $D=$ $1+\boldsymbol{\pi}^{2} / 4 f_{\pi}^{2}$ and $f_{\pi}=93 \mathrm{MeV}$ the pion decay constant, $N=(p n)^{T}$ is a heavy-nucleon field of velocity $v^{\mu}$ and $\operatorname{spin} S^{\mu}\left(S^{\mu}=(0, \vec{\sigma} / 2)\right.$ in the nucleon rest frame where $\left.v^{\mu}=(1, \overrightarrow{0})\right)$, $D_{\mu}$ is the covariant derivative, with $\left(D_{\mu}^{(\pi)}\right)_{a b}=D^{-1}\left(\delta_{a b} \partial_{\mu}+i e \epsilon_{3 a b} A_{\mu}\right)$ for a pion and $D_{\mu}^{(N)}=\partial_{\mu}+i \boldsymbol{\tau} \cdot\left(\boldsymbol{\pi} \times D_{\mu} \boldsymbol{\pi}\right) / 4 f_{\pi}^{2}-i e A_{\mu}\left(1+\tau_{3}\right) / 2$ for a nucleon, and "..." stands for other interactions with more pions, nucleons and/or delta fields that are not explicitly needed in the following. Note that the pion mass term comes from the explicit breaking of chiral symmetry by the average quark mass $\bar{m}=\left(m_{u}+m_{d}\right) / 2$, so $m_{\pi}^{2}=\mathcal{O}\left(M_{Q C D} \bar{m}\right)$. Isospin breaking from the quark mass difference, which is proportional to $\bar{m} \varepsilon=\mathcal{O}\left(m_{\pi}^{2} \varepsilon / M_{Q C D}\right)$ with $\varepsilon=\left(m_{d}-m_{u}\right) /\left(m_{u}+m_{d}\right) \simeq 1 / 3$, does not appear at this order. Note also that at this order the nucleon is static and couples only to longitudinal photons. Kinetic corrections and magnetic couplings have relative size $\mathcal{O}\left(Q / M_{Q C D}\right)$ and appear in $\mathcal{L}_{\text {str } / \text { em }}^{(1)}$. The same is true for the delta isobar, including the nucleon-delta transition through coupling to a transverse photon. The pion-nucleon coupling in Eq. (2) is not determined from symmetry, but is expected to be $\mathcal{O}(1)$, and indeed $g_{A}=1.267$. The GoldbergerTreiman relation $g_{A} m_{N}=f_{\pi} g_{\pi N N}$ holds in lowest order. A term in $\mathcal{L}_{\text {str } / \text { em }}^{(2)}$ provides an $\mathcal{O}\left(\left(m_{\pi} / M_{Q C D}\right)^{2}\right)$ correction that removes the so-called Goldberger-Treiman discrepancy.

In addition, we need $P$ - and $T$-violating interactions. The exact form of these interactions will depend on the mechanism of $C P$ violation. Just above $M_{Q C D}, T$-violating interactions among quarks and gluons can be classified through their scale dimensions, starting with the $\bar{\theta}$ term. In a basis where the quark fields $q=(u d)^{T}$ have been appropriately rotated [10], we can write

$$
\mathcal{L}_{\overline{7}}^{Q C D}=m_{\star} \bar{\theta} \bar{q} i \gamma_{5} q+\ldots
$$


where $m_{\star}=m_{u} m_{d} /\left(m_{u}+m_{d}\right)=\bar{m}\left(1-\varepsilon^{2} / 4\right) / 2 \simeq \bar{m} / 2$. Here "..." represents higherdimension operators - such as the quark EDM and color EDM, the Weinberg three-gluon operator, and four-quark interactions - which we will neglect.

The $\bar{\theta}$ term generates $T$-violating interactions in the low-energy EFT. It is the fourth component of the $S O(4)$ vector $P=\left(\bar{q} \boldsymbol{\tau} q, \bar{q} i \gamma_{5} q\right)$, so it generates EFT interactions that transform as $T$-violating, fourth components of $S O(4)$ vectors made out of hadronic fields. The lowest-dimension operator of this type is

$$
\mathcal{L}_{T}^{(1)}=-\frac{\bar{g}_{0}}{D} \bar{N} \boldsymbol{\tau} \cdot \boldsymbol{\pi} N
$$

where $\bar{g}_{0}$ is an $I=0 T$-violating $\pi N N$ coupling. From dimensional analysis, $\bar{g}_{0}=$ $\mathcal{O}\left(m_{\star} \bar{\theta} / f_{\pi}\right)=\mathcal{O}\left(m_{\pi}^{2} \bar{\theta} / f_{\pi} M_{Q C D}\right)$. Because of the factor of $m_{\pi}^{2}$ implicit in $\bar{g}_{0}, \mathcal{L}_{\bar{f}}^{(1)}$ effectively has index $\Delta=1$.

Interactions with higher indices can be constructed in similar ways. T-violating nucleondelta transitions through pion emission, for example, involve at least one derivative, and are thus suppressed by at least $\mathcal{O}\left(Q / M_{Q C D}\right)$ relative to those stemming from the Lagrangian (4). Among the higher-order operators, particularly relevant here are short-range contributions to the nucleon EDM,

$$
\mathcal{L}_{\bar{F}}^{(3)}=\bar{N}\left(\tilde{d}_{0}+\tilde{d}_{1} \tau_{3}\right)\left(S_{\mu} v_{\nu}-S_{\nu} v_{\mu}\right) N F^{\mu \nu}
$$

where $\tilde{d}_{0}\left(\tilde{d}_{1}\right)$ is a short-range contribution to the isoscalar (isovector) EDM of the nucleon. From dimensional analysis, $\tilde{d}_{i}=\mathcal{O}\left(e m_{\star} \bar{\theta} / M_{Q C D}^{2}\right)=\mathcal{O}\left(e m_{\pi}^{2} \bar{\theta} / M_{Q C D}^{3}\right)$. Direct short-range contributions to the momentum dependence of the EDFF first appear in $\mathcal{L}_{7}^{(5)}$, being further suppressed by $\mathcal{O}\left(\left(Q / M_{Q C D}\right)^{2}\right)$.

Here we denote by $i J_{e d}^{\mu}$ the $T$-violating nucleon current that interacts with the electron current $-i e \bar{e} \gamma^{\mu} e$ via the photon propagator $i D_{\mu \nu}=-i\left(\eta_{\mu \nu} / q^{2}+\ldots\right)$ to produce a contribution

$$
i T=-i e \bar{e}\left(k^{\prime}\right) \gamma^{\mu} e(k) D_{\mu \nu}(q) \bar{N}\left(p^{\prime}\right) J_{e d}^{\nu}(q) N(p),
$$

to the electron-nucleon $S$ matrix. We have $q^{2}=\left(p-p^{\prime}\right)^{2} \equiv-Q^{2}<0$. We can write

$$
J_{e d}^{\mu}(q)=2\left(F_{D}^{(0)}\left(-q^{2}\right)+F_{D}^{(1)}\left(-q^{2}\right) \tau_{3}\right)\left(S^{\mu} v \cdot q-S \cdot q v^{\mu}\right),
$$

where $F_{D}^{(0)}\left(Q^{2}\right)\left(F_{D}^{(1)}\left(Q^{2}\right)\right)$ is the isoscalar (isovector) EDFF of the nucleon, with $F_{D}^{(i)}(0)=$ $d_{i}$ the corresponding EDM.

When we consider the sizes of specific contributions to the neutron EDFF, the first tree-level contribution comes from the vertex generated by the Lagrangian of Eq. (5). The lowest-order one-loop graphs are built out of one vertex from the $T$-violating Lagrangian (4) and all other vertices from the strong Lagrangian (2). The one-loop diagrams that a priori could contribute to the EDFF are shown in Fig. 1. These one-loop diagrams contribute $\mathcal{O}\left(e \bar{g}_{0} f_{\pi} Q /\left(4 \pi f_{\pi}\right)^{2}\right)$ to $J_{e d}^{\mu}(q)$, and thus give rise to an EDFF of the same order as the short-range, tree-level contribution to the EDM, $\mathcal{O}\left(e m_{\star} \bar{\theta} / M_{Q C D}^{2}\right)$. 


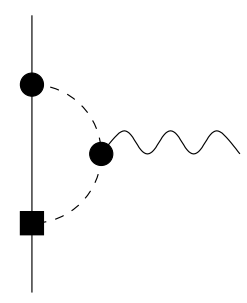

(a)

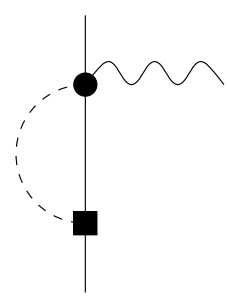

(b)

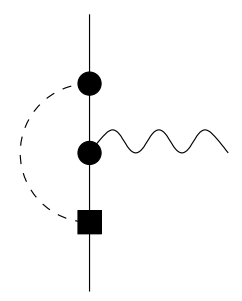

(c)

Figure 1: One-loop diagrams contributing to the nucleon electric dipole form factor in leading order. Solid, dashed and wavy lines represent nucleon, pion and photon, respectively; circles and squares stand for interactions from $\mathcal{L}_{\text {str } / e m}^{(0)}$ and $\mathcal{L}_{f}^{(1)}$, respectively. For simplicity only one of two possible orderings is shown here.

Because of the isospin structure, the contribution from Fig. 1(c) vanishes. We evaluate diagrams in Fig. 1 at $v \cdot q=0$, as a consequence of the static nature of the nucleon in leading order. Diagram 1(b) then also vanishes. Diagram 1(a) gives a non-zero contribution only to the isovector component of the nucleon EDFF.

The contributions from Fig. 1 contain divergent pieces that can be absorbed by redefining the tree-level contribution to the EDFF. Taking the tree and one-loop contributions, we have

$$
\begin{aligned}
& d_{0}=\tilde{d}_{0} \\
& d_{1}=\tilde{d}_{1}+\frac{e g_{A} \bar{g}_{0}}{8 \pi^{2} f_{\pi}}\left[\bar{\Delta}+2 \ln \frac{\mu}{m_{\pi}}\right],
\end{aligned}
$$

where $\mu$ is the renormalization scale introduced by dimensional regularization, and $\bar{\Delta} \equiv$ $2 / \varepsilon-\gamma_{E}+\ln 4 \pi(\varepsilon=4-d$, with $d$ the spacetime dimension). Using the GoldbergerTreiman relation and setting $\mu$ to $m_{N}$, the piece in Eq. (9) that is non-analytic in $m_{\pi}^{2}$ can be written as $\left(e g_{\pi N N} \bar{g}_{0} / 4 \pi^{2} m_{N}\right) \ln \left(m_{N} / m_{\pi}\right)$, in agreement with the result of Crewther et al. [5]. The short- and long-range contributions are in general of the same size, but a cancellation is unlikely due to the $\ln m_{\pi}$ dependence of the pion contribution. Note that short- and long-range physics cannot be separated with a measurement of the neutron $\mathrm{EDM} d_{n}=d_{0}-d_{1}$ alone.

Because the form factor is given by lowest-order loop graphs, it depends on the combination $Q^{2} / m_{\pi}^{2}$ only. Because the only non-vanishing contribution to this order comes from Fig. 1(a), the combination is actually $Q^{2} /\left(2 m_{\pi}\right)^{2}$. The EDFF defined in Eq. (7) is found to be given by

$$
\begin{aligned}
& F_{D}^{(0)}\left(Q^{2}\right)=d_{0} \\
& F_{D}^{(1)}\left(Q^{2}\right)=d_{1}-\frac{e g_{A} \bar{g}_{0}}{12 \pi^{2} f_{\pi}} F\left(\frac{Q^{2}}{\left(2 m_{\pi}\right)^{2}}\right)
\end{aligned}
$$




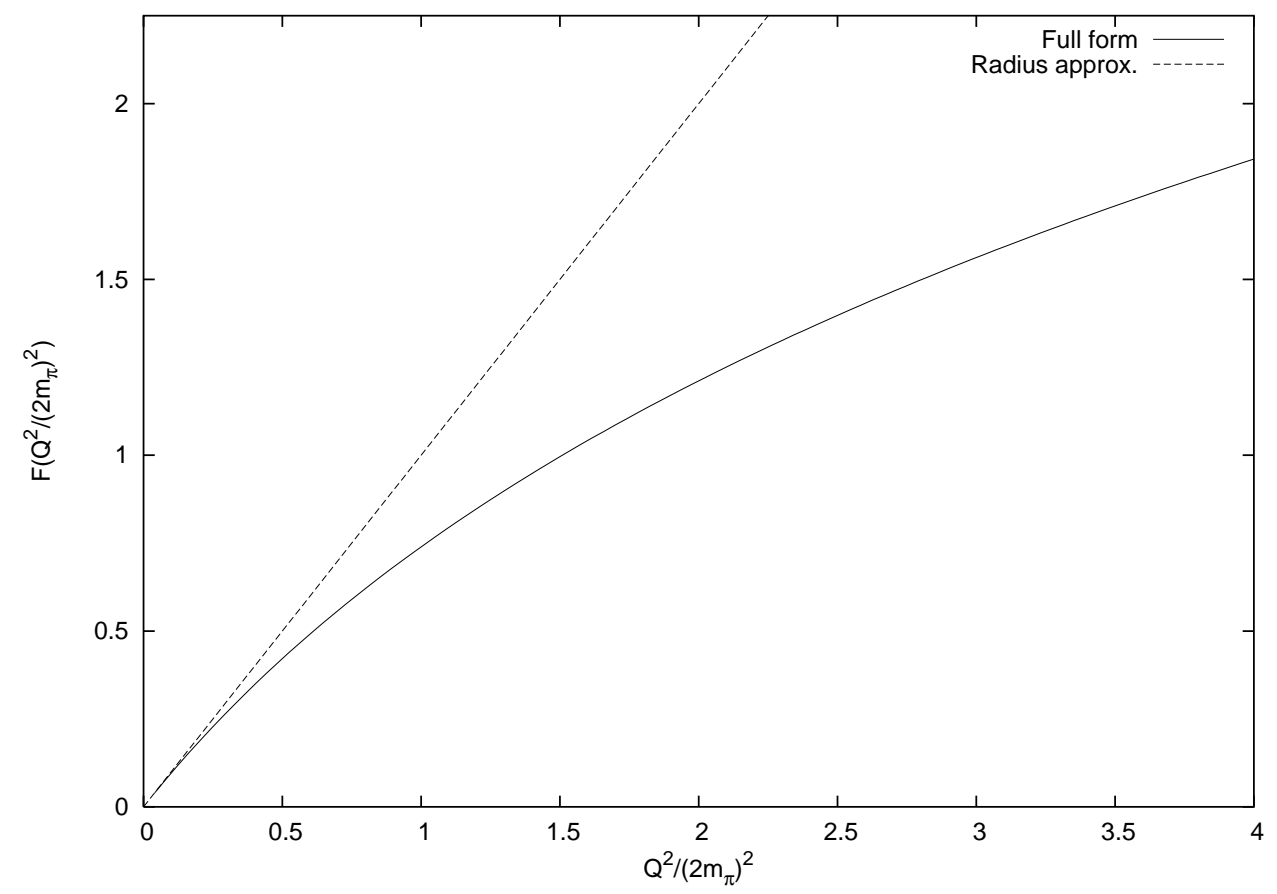

Figure 2: The function $F\left(Q^{2} /\left(2 m_{\pi}\right)^{2}\right)$ that enters the isovector electric dipole form factor $F_{D}^{(1)}: \chi \mathrm{PT}$ in leading order, Eq. (12) (solid line); and quadratic approximation, Eq. (13) (dotted line).

where

$$
F(x)=3\left\{\frac{1}{2} \sqrt{1+\frac{1}{x}} \ln \left(\frac{\sqrt{1+1 / x}+1}{\sqrt{1+1 / x}-1}\right)-1\right\} .
$$

Note that $F(0)=0$, so indeed this does meet the standard of being a form factor. The function $F$ is a testable prediction of $\chi \mathrm{PT}$ : it is plotted as a function of $Q^{2}$ in Fig. 2 .

For $x \ll 1$ we can expand $F(x)$ in powers of $x$,

$$
F(x)=x+\mathcal{O}\left(x^{2}\right) .
$$

This approximation is compared to the full functional form (12) in Fig. 2. The variation of the form factor with $Q$ can be characterized at very small momenta by the isovector EDM square radius (defined in analogy to the charge square radius),

$$
\left\langle r_{e d}^{2}\right\rangle^{(1)}=-6\left(\frac{d F_{D}^{(1)}}{d Q^{2}}\right)_{Q^{2}=0}=\frac{e g_{A} \bar{g}_{0}}{8 \pi^{2} f_{\pi}} \frac{1}{m_{\pi}^{2}} .
$$

While the EDM vanishes in the chiral limit, the radius of the EDFF is finite. As was noted in Ref. [9], $S^{\prime}=\left\langle r_{e d}^{2}\right\rangle^{(1)} / 6=e g_{A} \bar{g}_{0} / 48 \pi^{2} f_{\pi} m_{\pi}^{2}\left(-S^{\prime}\right)$ is the leading electromagnetic contribution to the Schiff moment of the the proton (neutron). 
The mass scale governing the variation of $F\left(Q^{2} /\left(2 m_{\pi}^{2}\right)\right)$ is in fact $2 m_{\pi}$, since it is generated by the pion cloud. The relative importance of the $Q^{2}$ variation of $F_{D}^{(1)}\left(Q^{2}\right)$ depends in addition on the ratio $e g_{A} \bar{g}_{0} / 12 \pi^{2} f_{\pi} d_{1}$. This ratio is a constant in the chiral limit. Since we expect it to be $\mathcal{O}(1)$, it is likely that $m_{\pi}$ sets the scale of the variation of $F_{D}^{(1)}\left(Q^{2}\right)$ as well. Unlike other nucleon form factors, a dipole approximation with a mass scale close to the rho-meson mass, $m_{\rho}$, should not be good even as a numerical approximation for the EDFF.

All other contributions to the EDFF are formally of higher order. Next-order effects come from diagrams with one insertion of an operator with one more derivative. First, there are operators in

$$
\begin{aligned}
\mathcal{L}_{\text {str } / e m}^{(1)}=\frac{1}{2 m_{N}}\left\{\begin{array}{l} 
\\
\bar{N}
\end{array}\left((v \cdot D)^{2}-D^{2}\right) N+\frac{g_{A}}{f_{\pi}}[i \bar{N}(v \cdot D \boldsymbol{\tau} \cdot \boldsymbol{\pi}) S \cdot D N+\text { H.c. }]\right. \\
\left.\quad+\frac{e}{2} \varepsilon_{\mu \nu \rho \sigma} \bar{N}\left(1+\kappa_{0}+\left(1+\kappa_{1}\right) \tau_{3}\right) v^{\mu} S^{\nu} N F^{\rho \sigma}+\ldots\right\}+\ldots
\end{aligned}
$$

These are nucleon recoil corrections to the static limit and photon coupling to the nucleon magnetic moment (including the isoscalar and isovector anomalous magnetic moments, $\kappa_{0}=-0.12$ and $\kappa_{1}=3.7$ respectively). Second, there is a new operator in

$$
\mathcal{L}_{T}^{(2)}=\frac{2 \bar{h}_{0}}{D} \boldsymbol{\pi} \cdot D_{\mu} \boldsymbol{\pi} \bar{N} S^{\mu} N
$$

with an undetermined coefficient $\bar{h}_{0}=\mathcal{O}\left(m_{\star} \bar{\theta} / f_{\pi}^{2} M_{Q C D}\right)=\mathcal{O}\left(m_{\pi}^{2} \bar{\theta} / f_{\pi}^{2} M_{Q C D}^{2}\right)$. The corresponding contributions to the nucleon EDFF are also calculable, as they are still given by long-range physics associated with the pion cloud. Although the strong-interaction corrections depend on known parameters, Eq. (16) introduces a new $T$-violating parameter. Both types of contributions - from Eqs. (15) and (16) - are suppressed by $\mathcal{O}\left(Q / M_{Q C D}\right)$ relative to the contributions calculated here.

Note that if $\mathcal{L}_{7}$ contained other non-derivative, $T$-violating pion-nucleon interactions, $\pi_{3} \bar{N} N$ and $\pi_{3} \bar{N} \tau_{3} N$ [11], our previous results would remain unchanged. However, the chiral-symmetry transformation properties of the $\bar{\theta}$ term do not yield such terms.

In conclusion, we have presented results for the electric dipole form factor of the nucleon due to the $\bar{\theta}$ term in leading order in $\chi \mathrm{PT}$. We have shown that the the EDFF is isovector, with a $Q^{2}$ dependence determined by a non-derivative $T$-violating pion-nucleon coupling and the pion mass, see Eqs. (11) and (12), and Fig. 2. Under the assumption that higher-order results are not afflicted by anomalously-large dimensionless factors, the error of our results at momentum $Q$ should be $\sim Q / m_{\rho}$.

\section{Acknowledgments}

We thank Steve Puglia for discussions and the Institute for Nuclear Theory at the University of Washington for hospitality at the beginning of this work. This research was supported in part by the US Department of Energy (WHH, UvK) and the Alfred P. Sloan Foundation $(\mathrm{UvK})$. 


\section{References}

[1] I.B. Khriplovich and S.K. Lamoreaux, CP Violation Without Strangeness (Springer Verlag, Berlin, 1997).

[2] P.G. Harris et al., Phys. Rev. Lett. 82, 904 (1999).

[3] S. Weinberg, The Quantum Theory of Fields, Vol. 2 (Cambridge Univ. Pr., Cambridge, 1996).

[4] V. Bernard, N. Kaiser, U.-G Meißner, Int. J. Mod. Phys. E4 (1995) 193.

[5] R.J. Crewther, P. Di Vecchia, G. Veneziano, and E. Witten, Phys. Lett. B 88, 123 (1979); (E) 91, 487 (1980).

[6] H.-Y. Cheng, Phys. Rev. D 44, 166 (1991); A. Pich and E. de Rafael, Nucl. Phys. B 367, 313 (1991) P. Cho, Phys. Rev. D 48, 3304 (1993); B. Borasoy, Phys. Rev. D 61, 114017 (2000).

[7] V. Bernard, N. Kaiser, J. Kambor, and U.-G. Meißner, Nucl. Phys. B 388, 315 (1992); V. Bernard, H.W. Fearing, T.R. Hemmert, and U.-G. Meißner, Nucl. Phys. A 635, 121 (1998), (E) 642, 563 (1998).

[8] C.M. Maekawa and U. van Kolck, Phys. Lett. B 478, 73 (2000); C.M. Maekawa, J.S. Veiga, and U. van Kolck, Phys. Lett. B 488, 167 (2000).

[9] S. Thomas, Phys. Rev. D 51, 3955 (1995).

[10] V. Baluni, Phys. Rev. D 19, 2227 (1979).

[11] G. Barton, Nuovo Cim. 19, 512 (1961). 